\title{
Azithromycine et pathologies bronchiques chroniques : Quels patients ? Quels bénéfices ? Quelles modalités?
}

\section{Azithromycine and chronic bronchial diseases: For which patients? For which benefits? With what modalities?}

\author{
P. Pronost ${ }^{\text {a }}$, C. Tromeur ${ }^{\text {a,b,c }}$
}

(a) Département de médecine interne et Pneumologie, Hôpital de la Cavale Blanche, CHRU Brest.

(b) Groupe d'Etude de la Thrombose de Bretagne Occidentale, Equipe d'Accueil 3878, CHRU de Brest

(c) Centre d'Investigation Clinique INSERM 1412, Université de Bretagne Occidentale, Brest

\author{
Auteur correspondant : \\ Dr Pauline Pronost \\ Département de médecine interne et Pneumologie \\ boulevard Tanguy Prigent \\ 29200 Brest
}

Courriel : paulinepronost@orange.fr 


\section{RESUME}

L'azithromycine est un macrolide de plus en plus utilisé pour ses propriétés anti-inflammatoires dans les pathologies bronchiques chroniques. Ce traitement peut être proposé à des patients atteints de dilatation des bronches, d'asthme et de bronchopneumopathie chronique obstructive sévère ayant eu plus de 3 exacerbations par an ou ayant eu une dégradation de leur fonction respiratoire malgré un traitement de fond optimal bien conduit. Malgré l'efficacité sur le nombre d'exacerbations, l'utilisation des macrolides au long cours doit faire l'objet de précaution du fait de ses toxicités cardiovasculaires, otologiques et de l'émergence de bactéries résistantes. De plus, les études restent insuffisantes pour établir la posologie optimale ainsi que la durée des traitements par azithromycine.

\section{MOTS CLES}

Azithromycine, DDB (dilatation de bronche), BPCO (bronchopneumopathie chronique obstructive), exacerbations. 


\begin{abstract}
Azithromycin is a macrolide widely used in chronic bronchial diseases due to its anti-inflammatory properties. This treatment is prescribed to patients with bronchiectasis, asthma and severe chronic obstructive pulmonary disease who present more than 3 exacerbations per year or a deterioration of respiratory function despite an optimal treatment. Macrolides decrease the number of exacerbation but azythromycine must be prescribed carefully. Indeed, it involves potential cardiovascular and otological toxicities and the emergence of resistant bacteria. In addition, studies remain insufficient to establish the optimal dosage and duration of azithromycine.
\end{abstract}

\title{
KEY WORDS
}

Azithromycin, bronchiectasis, chronic obstructive pulmonary disease (COPD), exacerbations 


\section{Introduction}

Les macrolides sont connus depuis plusieurs années pour leurs propriétés anti-inflammatoires et immunomodulatrices en plus de leur activité antimicrobienne (1). Ils ont aussi des propriétés «non antibiotiques » sur des bactéries naturellement résistantes comme le Pseudomonas aeruginosa. Leur intérêt a été démontré depuis presque un demi-siècle dans la panbronchiolite diffuse touchant quasi exclusivement la population asiatique. Depuis, leur utilisation s'est développée dans d'autres maladies respiratoires chroniques $(1,2)$. En effet, des études cliniques ont montré une certaine efficacité des macrolides dans des maladies bronchiques dont la part inflammatoire est importante, telles que la bronchopneumopathie chronique obstructive (BPCO) et la dilatation de bronche (DDB) $(3,4)$. Toutefois, l'utilisation prolongée de ces traitements doit se faire avec précaution. Leur prescription est de plus en plus répandue et se fait parfois sans tenir compte des effets secondaires potentiellement délétères, alors même que l'indication retenue de l'azithromycine n'est pas adaptée au patient et à sa pathologie respiratoire.

Notre objectif est de faire une mise au point sur les indications et les effets secondaires de l'azithromycine dans les pathologies bronchiques chroniques (hors mucoviscidose).

\section{Indications}

\subsection{Indication dans la BPCO}

Deux études randomisées contrôlées ont permis de montrer que l'administration d'azithromycine ( $250 \mathrm{mg} / \mathrm{j}$ ou $500 \mathrm{mg}$ trois fois par semaine) chez des patients atteints de BPCO modérée à sévère (stade 2 à 4 de la classification de GOLD) permettait de diminuer le taux d'exacerbations et d'augmenter le délai d'apparition de la première exacerbation (3,5). La première étude multicentrique incluant 559 patients dans le groupe placebo et 558 dans le groupe azithromycine $(250 \mathrm{mg} / \mathrm{jour})$ montrait une réduction significative du nombre d'exacerbations par patients par an dans le groupe azithromycine ( 1,48 versus 1,$83 ; \mathrm{p}=0,01)$ et du délai d'apparition de la première exacerbation dans le groupe azithromycine par rapport au groupe placebo (174 jours versus 266 jours ; $p<0,001)$ (3). Uzun et al. ont réalisé une étude prospective de 92 patients (47 patients dans le groupe azithromycine et 45 patients dans le groupe placebo) évaluant l'efficacité de l'azithromycine $500 \mathrm{mg}$ trois fois par semaine sur 1 an (5). Cette étude a montré une diminution significative du nombre d'exacerbations dans le groupe azithromycine (84 versus 129 exacerbations dans le groupe placebo; $p<0,001)$ et du délai d'apparition de la première exacerbation (130 jours dans le groupe azithromycine contre 59 jours dans le groupe placebo; $\mathrm{p}=0,001$ ). Enfin, une étude récente a évalué 109 patients atteints de BPCO sévères traités par azithromycine (500 mg trois fois par semaine) sur une période de 24 à 36 mois (6). Cette étude rétrospective a montré une réduction du nombre d'hospitalisations et du nombre d'exacerbations de plus de 50\% avec peu d'effets indésirables. Toutefois, l'efficacité semble diminuée au-delà de deux ans, expliquée en partie, par l'émergence de souches bactériennes résistantes. Aucune étude n'a montré d'amélioration sur les paramètres ventilatoires ni sur la qualité de vie. Compte tenu 
des effets connus délétères des exacerbations de BPCO sur la diminution des capacités respiratoires, toute mesure thérapeutique visant à en réduire le nombre doit être considérée comme bénéfique, justifiant la prescription d'un tel traitement au long cours. Les recommandations de la société Française de pneumologie de 2016 proposent un traitement préventif par l'azithromycine $250 \mathrm{mg} /$ jour chez les BPCO sévère (oxygénothérapie au long cours, au moins trois exacerbations par an malgré un traitement inhalé optimal) diminuant le risque d'exacerbations sur 3 ans et allongeant le délai de la première exacerbation (7). Le tableau 1 illustre les principales études évaluant l'azithromycine à visée anti-inflammatoire chez les patients atteints de BPCO.

\subsection{Indication dans la dilatation des bronches (DDB)}

L'azithromycine est recommandé chez les patients de plus de 6 ans atteints de mucovisidose avec DDB colonisés à Pseudomonas aeruginosa afin d'améliorer la fonction respiratoire et de diminuer le nombre d'exacerbations (8). En dehors de la mucoviscidose, l'utilisation de l'azithromycine reste controversée dans le cadre de bronchiectasies.

En effet, deux études randomisées, contrôlées, ont montré une diminution significative du nombre d'exacerbations sous macrolides au long cours $(4,9)$. Altenburg et al. ont réalisé une étude chez 83 patients (40 patients dans le groupe azithromycine et 43 patients dans le groupe placebo) évaluant l'efficacité de l'azithromycine $250 \mathrm{mg} /$ jour sur 1 an. Cette étude a montré une diminution significative du nombre d'exacerbations par patient par an dans le groupe azithromycine $(0,84$ versus 2,05; p <0,001) (9). En revanche, cette étude n'a montré aucun effet évident sur la qualité de vie et la fonction respiratoire. De plus, le niveau de gravité et le profil évolutif des DDB ne sont pas informés, constituant une certaine limite à leur interprétation. De plus, les critères utilisés dans la BPCO pour définir les exacerbations n'incluent pas la toux, symptôme dominant chez les patients atteints de DDB. Il est ainsi difficile de déterminer avec précision quels patients atteints de DDB tireraient un bénéfice d'un traitement au long cours par macrolides compte tenu de l'hétérogénéité des profils de cette population. L'utilisation de macrolides doit être réservée aux patients atteints de DDB avec exacerbations fréquentes et réfractaires à une prise en charge standard. Le tableau 2 illustre les principales études évaluant les macrolides chez les patients atteints de bronchectasies.

\subsection{Indication dans l'asthme}

Une méta-analyse Cochrane a montré une légère diminution de l'hyperréactivité bronchique sans augmentation du volume expiratoire maximal par seconde (VEMS) et sans de réels bénéfices des macrolides chez les patients atteints d'asthme (10). Cependant, des travaux récents relancent l'intérêt potentiel des macrolides dans cette pathologie. Un essai randomisé (étude AZISAST) incluant 109 patients asthmatiques exacerbateurs (55 patients dans le groupe azithromycine et 54 dans le groupe placebo) montrait que la prise d'azithromycine (250 mg trois jours par semaine) pendant 6 mois ne permettait pas de réduire le nombre d'exacerbations, ni d'améliorer les paramètres 
fonctionnels respiratoires, ni la qualité de vie des patients asthmatiques sévères (11). En revanche, une réduction significative du taux d'exacerbations par rapport au placebo était observée, dans un sousgroupe de patients dont le taux d'éosinophiles sanguin était inférieur à $200 / \mathrm{mm}^{3}(\mathrm{p}=0,013)$. Enfin, une dernière étude (AMAZES) incluant 420 patients asthmatiques non contrôlés a montré un effet favorable significatif en faveur de l'azithromycine $500 \mathrm{mg}$ trois fois par semaine versus placebo pendant 48 semaines sur la réduction du nombre d'exacerbations et sur l'amélioration de la qualité de vie $(\mathrm{p}<0,0001)$ (12). Le tableau 3 illustre les principales études évaluant les macrolides chez les patients atteints d'asthme.

\subsection{Indication dans les autres pathologies pulmonaires}

L'utilisation de l'azithromycine administrée à la dose de $250 \mathrm{mg}$ trois fois par semaine retarde l'apparition de la bronchiolite oblitérante post-transplantation pulmonaire et s'accompagne d'une diminution de l'inflammation des voies aériennes (13). Les macrolides ont aussi été évalués, sans succès, dans d'autres pathologies comme la pneumonie organisée cryptogénique ou dans des pathologies rares comme la fibrose pulmonaire (2). Ainsi, en dehors des pathologies précédemment décrites, il n'y a pas d'indication à l'utilisation de macrolides au long cours.

\section{Effets secondaires}

Des effets secondaires courants sont observés en cas d'instauration de l'azithromycine (diarrhée, nausées, vomissements, douleurs abdominales, hépatites cytolytiques). Toutefois, certaines toxicités potentielles doivent faire l'objet d'une attention particulière.

\subsection{Toxicité cardiovasculaire}

Tous les macrolides présentent un risque d'allongement de l'intervalle QT en bloquant le canal potassique des cellules myocardiques. De plus, métabolisés par le cytochrome P450, ils interfèrent avec de nombreux médicaments arythmogènes. Contrairement aux autres macrolides, l'azithromycine n'est pas métabolisé par le cytochrome P450, impliquant ainsi un faible risque d'arythmie ventriculaire. L'allongement du QTC et le risque de torsade de pointe surviennent chez des patients présentant des facteurs de risque coexistants (14). En effet, Poluzzi et al. ont étudié les cas d'arythmie induit par les médicaments entre 2004 et 2011 : sur 63 cas de torsade de pointe liés à l'azithromycine, $59 \%$ des patients recevaient déjà des thérapeutiques allongeant l'intervalle QT (15). Bien que le risque de décès d'origine cardiovasculaire secondaire à la prise d'azithromycine soit faible (1 sur 20 000), le risque est beaucoup plus élevé (environ 1 personne sur 4 000) chez les patients déjà atteints de maladie cardiaque ainsi qu'en cas de traitement au long cours (16,17). Ainsi, les comorbidités cardiovasculaires étant fréquentes chez les patients atteints de BPCO, la prescription de macrolides doit se faire avec prudence. 


\subsection{Toxicité otologique}

La toxicité otologique touchant principalement les fréquences liées à la parole est dose dépendante. Quelques cas irréversibles ont été décrits dans la littérature médicale (18). Toutefois, cette atteinte a été observée principalement en cas de fortes posologies, et concernerait peu les doses «anti inflammatoires ». Albert et al. ont décrit une baisse significative de l'audition chez $25 \%$ des patients ayant reçu une posologie d'azithromycine de $250 \mathrm{mg}$ par jour pendant 1 an contre $20 \%$ dans le groupe placebo $(\mathrm{p}=0,04)$ (3). D'autre part, un tiers des patients a normalisé son audiogramme que le traitement par azithromycine ait été poursuivi ou non. Toutefois, il semble difficile de ne pas restreindre la prescription de macrolides chez les patients avec des troubles auditifs préexistants ou de poursuivre le traitement en cas d'apparition de troubles auditifs.

\subsection{Impact sur la flore bactérienne}

L'administration prolongée de faibles doses d'azithromycine soulève la question de l'apparition rapide et persistante de souches bactériennes résistantes. En effet, l'émergence de souches d'Haemophilus influenzae (19), de staphylococcus aureus (20,21) de streptococcus pneumoniae (21) et de streptococcus milleri (22) ont été décrites. Dans les études randomisées dans la DDB, le taux de résistance des germes pathogènes est passé de 35 à $88 \%$ chez les patients traités par azithromycine (23). Albert et al. ont montré que la proportion de bactéries résistantes aux macrolides ayant colonisé le nasopharynx au cours de l'étude était de $81 \%$ dans le groupe azithromycine contre $41 \%$ dans le groupe placebo (3). Ainsi, l'impact sur la flore bactérienne n'est pas négligeable et impose un respect strict des indications de l'azithromycine au long cours dans les pathologies bronchiques chroniques. D'autre part, l'apparition des bactéries résistantes imposent de ne pas poursuivre ce traitement s'il s'avère non efficace sur le nombre d'exacerbations.

\section{En pratique}

L'azythromycine au long cours peut être proposé à des patients atteints de BPCO ou de DDB ayant plus de 3 exacerbations l'année précédente ou ayant une dégradation de leur fonction respiratoire malgré un traitement de fond bien conduit (7). Ce traitement semble aussi être utile chez les patients asthmatiques dont le traitement inhalé est optimal et qui présentent de nombreuses exacerbations. Ce traitement ne remplace pas les traitements de fond des pathologies bronchiques chroniques et doit être prescrit à la condition d'un traitement optimal bien conduit non efficace.

Pour les patients atteints de DDB et de BPCO, l'azithromycine peut être utilisée à la dose de $250 \mathrm{mg} /$ jour ou $250 \mathrm{mg}$ (moins de $55 \mathrm{~kg}$ ) à $500 \mathrm{mg}$ (plus de $55 \mathrm{~kg}$ ) trois fois par semaine (7). Les patients asthmatiques exacerbateurs fréquents dont le traitement inhalé est optimal, peuvent bénéficier d'azithromycine $500 \mathrm{mg}$ trois fois par semaine. Le traitement doit être poursuivi tant que le bénéfice sur la fréquence des exacerbations est maintenu. = 
Le traitement par macrolide au long cours ne doit pas être proposé en cas de pathologie cardiaque évoluée, d'intervalle QT $>450 \mathrm{~ms}$, de traitement pouvant allonger l'intervalle QT, de tachycardie $>100 /$ min (17). Ainsi, une surveillance clinique, avec en particulier la recherche de troubles auditifs et électrocardiographique peut être proposée tous les trois mois. Il est aussi recommandé de s'assurer de l'absence de mycobactéries atypiques dans les cultures des expectorations avant d'envisager un traitement prolongé par macrolides pour éviter un traitement inadapté et l'acquisition de résistance bactérienne (24). L'objectif est de diminuer le nombre d'exacerbations.

\section{Conclusion}

Actuellement, les macrolides administrés au long cours dans un but anti-inflammatoire sont proposés à des patients atteints de DDB ou de BPCO sans comorbidité cardiovasculaire évoluée présentant des exacerbations fréquentes et/ou dont l'état respiratoire se dégrade rapidement malgré une prise en charge initiale optimale. Ils sont aussi de plus en plus proposés aux patients asthmatiques exacerbateurs fréquents. Leur efficacité a été démontrée sur la fréquence des exacerbations. Compte tenu des conséquences des exacerbations aiguës (risque de décès, altération de la qualité de vie et de la fonction respiratoire et le coût des soins), l'utilisation de l'azithromycine est une option thérapeutique valable pour les patients atteints de pathologies bronchiques chroniques non contrôlées par un traitement optimal usuel. Toutefois, les indications de prescription de l'azithromycine doivent être respectées en prenant compte des effets secondaires potentiels. De plus, peu de données sont disponibles sur la durée optimale des macrolides au long cours, l'efficacité de ce traitement ayant été évaluée majoritairement sur le court terme.

Conflits d'intérêt : les auteurs déclarent ne pas avoir de conflits d'intérêt avec cet article. 


\section{Références}

1. Zarogoulidis P, Papanas N, Kioumis I, Chatzaki E, Maltezos E, Zarogoulidis K. Macrolides: from in vitro anti-inflammatory and immunomodulatory properties to clinical practice in respiratory diseases. Eur J Clin Pharmacol 2012;68:479-503.

2. Spagnolo P, Fabbri LM, Bush A. Long-term macrolide treatment for chronic respiratory disease. Eur Respir J 2013;42:239-51.

3. Albert RK, Connett J, Bailey WC, Casaburi R, Cooper JAD, Criner GJ, et al. Azithromycin for Prevention of Exacerbations of COPD. N Engl J Med 2011;365:689-98.

4. Wong C, Jayaram L, Karalus N, Eaton T, Tong C, Hockey H, et al. Azithromycin for prevention of exacerbations in non-cystic fibrosis bronchiectasis (EMBRACE): a randomised, double-blind, placebo-controlled trial. Lancet 2012;380:660-7.

5. Uzun S, Djamin RS, Kluytmans JAJW, Mulder PGH, van't Veer NE, Ermens AAM, et al. Azithromycin maintenance treatment in patients with frequent exacerbations of chronic obstructive pulmonary disease (COLUMBUS): a randomised, double-blind, placebo-controlled trial. Lancet Respir Med 2014;2:361-8.

6. Pomares X, Montón C, Bullich M, Cuevas O, Oliva JC, Gallego M, et al. Clinical and Safety Outcomes of Long-Term Azithromycin Therapy in Severe COPD Beyond the First Year of Treatment. Chest 2018;153:1125-33.

7. Zysman M, Chabot F, Devillier P, Housset B, Morelot-Panzini C, Roche N. Pharmacological treatment optimization for stable chronic obstructive pulmonary disease. Proposals from the Société de Pneumologie de Langue Française. Rev Mal Respir 2016;33:911-36.

8. Flume PA, O'Sullivan BP, Robinson KA, Goss CH, Mogayzel PJ, Willey-Courand DB, et al. Cystic Fibrosis Pulmonary Guidelines: Chronic Medications for Maintenance of Lung Health. Am J Respir Crit Care Med 2007;176:957-69.

9. Altenburg J, de Graaff CS, Stienstra Y, Sloos JH, van Haren EHJ, Koppers RJH, et al. Effect of Azithromycin Maintenance Treatment on Infectious Exacerbations Among Patients With NonCystic Fibrosis Bronchiectasis: The BAT Randomized Controlled Trial. JAMA 2013;309:1251-9.

10. Ferrara G, Losi M, Franco F, Corbetta L, Fabbri LM, Richeldi L. Macrolides in the treatment of asthma and cystic fibrosis. Respir Med 2005;99:1-10.

11. Brusselle GG, VanderStichele C, Jordens P, Deman R, Slabbynck H, Ringoet V, et al. Azithromycin for prevention of exacerbations in severe asthma (AZISAST): a multicentre randomised double-blind placebo-controlled trial. Thorax 2013;68:322-9.

12. Gibson PG, Yang IA, Upham JW, Reynolds PN, Hodge S, James AL, et al. Effect of azithromycin on asthma exacerbations and quality of life in adults with persistent uncontrolled asthma (AMAZES): a randomised, double-blind, placebo-controlled trial. Lancet 2017;390:65968.

13. Vos R, Vanaudenaerde BM, Verleden SE, De Vleeschauwer SI, Willems-Widyastuti A, Van Raemdonck DE, et al. A randomised controlled trial of azithromycin to prevent chronic rejection after lung transplantation. Eur Respir J 2011;37:164-72.

14. Justo D, Zeltser D. Torsades de pointes induced by antibiotics. Eur J Intern Med 2006;17:254-9.

15. Poluzzi E, Raschi E, Motola D, Moretti U, De Ponti F. Antimicrobials and the Risk of Torsades de Pointes: The Contribution from Data Mining of the US FDA Adverse Event Reporting System. Drug Saf 2010;33:303-14.

16. Taylor SP, Sellers E, Taylor BT. Azithromycin for the Prevention of COPD Exacerbations: The Good, Bad, and Ugly. Am J Med 2015;128:1362.e1-6.

17. Ray WA, Murray KT, Hall K, Arbogast PG, Stein CM. Azithromycin and the risk of cardiovascular death. N Engl J Med 2012;366:1881-90.

18. Ress BD, Gross EM. Irreversible Sensorineural Hearing Loss as a Result of Azithromycin Ototoxicity: A Case Report. Ann Otol Rhinol Laryngol 2000;109(4):435-7.

19. Roberts MC, Soge OO, No DB. Characterization of macrolide resistance genes in Haemophilus influenzae isolated from children with cystic fibrosis. J Antimicrob Chemother 2011;66:100-4.

20. Hansen CR, Pressler T, Hoiby N, Johansen HK. Long-term, low-dose azithromycin treatment reduces the incidence but increases macrolide resistance in Staphylococcus aureus in Danish CF patients. J Cyst Fibros 2009;8:58-62. 
21. Prunier A-L, Malbruny B, Laurans M, Brouard J, Duhamel J-F, Leclercq R. High Rate of Macrolide Resistance in Staphylococcus aureus Strains from Patients with Cystic Fibrosis Reveals High Proportions of Hypermutable Strains. J Infect Dis 2003;187:1709-16.

22. Grinwis ME, Sibley CD, Parkins MD, Eshaghurshan CS, Rabin HR, Surette MG. Macrolide and Clindamycin Resistance in Streptococcus milleri Group Isolates from the Airways of Cystic Fibrosis Patients. Antimicrob Agents Chemother 2010;54:2823-9.

23. Serisier DJ, Martin ML, McGuckin MA, Lourie R, Chen AC, Brain B, et al. Effect of Long-term, Low-Dose Erythromycin on Pulmonary Exacerbations Among Patients With Non-Cystic Fibrosis Bronchiectasis: The BLESS Randomized Controlled Trial. JAMA 2013;309:1260-7.

24. Jouneau S, Desrues B. Macrolides au long cours et pathologie bronchique chronique de l'adulte : intérêts et limites. Presse Med 2014;43:510-9. 
TABLEAUX

Tableau 1 : Principales caractéristiques des études dans la BPCO.

\begin{tabular}{|c|c|c|c|c|c|c|c|c|}
\hline Auteur & Méthodologie & Intervention & Durée & $\begin{array}{c}\text { Critère } \\
\text { D'inclusion }\end{array}$ & $\begin{array}{l}\text { Critère de jugement } \\
\text { principal }\end{array}$ & $\begin{array}{c}\text { Critère de } \\
\text { jugement } \\
\text { secondaire }\end{array}$ & $\begin{array}{c}\text { Résultat sur le critère de jugement } \\
\text { principal }\end{array}$ & $\begin{array}{c}\text { Résultat sur autre } \\
\text { critère de jugement }\end{array}$ \\
\hline Albert et al. (3) & $\begin{array}{l}\text { Multicentrique } \\
\text { Randomisée } \\
\text { Contrôlée } \\
\text { Groupe parallèle } \\
\text { Double aveugle } \\
\text { Prospectif } \\
\mathrm{N}=1142 \text { patients } \\
\end{array}$ & $\begin{array}{l}\text { AZM } \\
250 \mathrm{mg} \text { par jour }\end{array}$ & 1 an & $\begin{array}{l}\text { BPCO sous oxygène de } \\
\text { longue durée ou ayant } \\
\text { reçu des stéroïdes } \\
\text { l'année précédente ou } \\
\text { hospitalisés pour } \\
\text { exacerbation }\end{array}$ & $\begin{array}{l}\text { Délai d'apparition de } \\
\text { la } 1 \text { ère exacerbation }\end{array}$ & $\begin{array}{l}\text { - spirométrie } \\
\text { - qualité de vie } \\
\text { - données } \\
\text { bactériologiques } \\
\text { - tolérance }\end{array}$ & $\begin{array}{l}\text { - Placebo }: \mathrm{n}=559 \\
1^{\text {ere }} \text { exacerbation }: 174 \text { jours } \\
\text { - AZM }: \mathrm{n}=558 \\
1^{\text {ere }} \text { exacerbation : } \\
266 \text { jours } \\
\mathrm{P}<0,001\end{array}$ & $\begin{array}{l}\text { Pas de différence sur } \\
\text { les critères secondaires }\end{array}$ \\
\hline $\begin{array}{l}\text { Uzun et al. } \\
\text { (5) }\end{array}$ & $\begin{array}{l}\text { Prospective } \\
\text { Randomisée } \\
\text { Contrôlée } \\
\text { Double aveugle } \\
\mathrm{N}=92 \text { patients }\end{array}$ & $\begin{array}{l}\text { AZM } \\
500 \text { mg trois fois par } \\
\text { semaine }\end{array}$ & 1 an & $\begin{array}{l}\text { BPCO sans exacerbation } \\
\text { dans le mois précédent }\end{array}$ & $\begin{array}{l}\text { Nombre } \\
\text { d'exacerbation et délai } \\
\text { d'apparition de la } 1^{\text {ère }} \\
\text { exacerbation }\end{array}$ & $\begin{array}{l}\text { - qualité de vie } \\
\text { - CRP } \\
\text { - tolérance }\end{array}$ & $\begin{array}{l}\text { - Placebo } n=45: 129 \text { exacerbations } \\
1^{\text {ere }} \text { exacerbation }: 59 \text { jours } \\
\text { - AZM n=47 : 84 exacerbations } \\
1^{\text {ere }} \text { exacerbation }: 130 \text { jours } \\
\mathrm{P}<0,001\end{array}$ & $\begin{array}{l}\text { Pas de différences } \\
\text { significatives sur les } \\
\text { critères secondaires }\end{array}$ \\
\hline $\begin{array}{l}\text { Pomares et al. } \\
\text { (6) }\end{array}$ & $\begin{array}{l}\text { Cohorte } \\
\text { Rétrospective } \\
109 \text { patients }\end{array}$ & $\begin{array}{l}\text { LT-CC-A : AZM } \\
500 \text { mg trois fois par } \\
\text { semaine pendant } \\
24 \text { mois } \\
\text { ST-CC-A : AZM } \\
500 \text { mg trois fois par } \\
\text { semaine pendant } \\
12 \text { mois }\end{array}$ & $\begin{array}{l}1 \text { an } \\
2 \text { ans } \\
3 \text { ans }\end{array}$ & $\begin{array}{l}\text { BPCO avec plus de } 4 \\
\text { exacerbations dans le } \\
\text { mois précédent }\end{array}$ & $\begin{array}{l}\text { Nombre } \\
\text { d'exacerbation et } \\
\text { nombre } \\
\text { d'hospitalisation }\end{array}$ & - tolérance & $\begin{array}{l}\text { - réduction du nombre } \\
\text { d'exacerbations : } 56,2 \% \text { à un an, } 70 \% \\
\text { à 2ans, } 41 \% \text { à } 36 \text { mois } \\
\text { - réduction du nombre } \\
\text { d'hospitalisations : } \\
62,5 \% \text { à un an, } 75,8 \% \text { à deux ans, } \\
39,8 \% \text { à } 36 \text { mois }\end{array}$ & $\begin{array}{l}\text { Aucun effet } \\
\text { indésirable grave }\end{array}$ \\
\hline
\end{tabular}

AZM : azithromycine ; CRP : C-réactive protéine ; VEMS : volume expiratoire maximale/seconde ; ATB : antibiotique ; TM6 : test de marche six minutes ; SGRQ : Saint George's respiratory questionnaire ; DDB : dilatation des bronches ; LT-CC-A : long term continous cyclic azithromycin ; STCCA : short term continous cyclic azithromycin 
Tableau 2 : Principales caractéristiques des études dans la dilatation des bronches (hors mucoviscidose).

\begin{tabular}{|c|c|c|c|c|c|c|c|c|}
\hline Auteur & Méthodologie & Intervention & Durée & Critère d'inclusion & $\begin{array}{c}\text { Critère de jugement } \\
\text { principal }\end{array}$ & Critère secondaires & $\begin{array}{c}\text { Résultats critères de jugement } \\
\text { principal }\end{array}$ & $\begin{array}{l}\text { Résultats sur critère } \\
\text { secondaires }\end{array}$ \\
\hline $\begin{array}{l}\text { Wong et al. } \\
\text { (4) }\end{array}$ & $\begin{array}{l}\text { Multicentrique } \\
\text { Randomisée } \\
\text { Contrôlée } \\
\text { Double aveugle } \\
141 \text { patients }\end{array}$ & $\begin{array}{l}\text { AZM } 500 \mathrm{mg} \\
3 \text { fois par semaine }\end{array}$ & 6 mois & $\begin{array}{l}\text { Au moins } 1 \text { exacerbation } \\
\text { l'année précédente avec } \\
\text { DDB confirmée au scanner }\end{array}$ & $\begin{array}{l}\text { Nombre d'exacerbations } \\
\text { dans les } 6 \text { premiers mois } \\
\text { - VEMS } \\
\text { - SGRQ }\end{array}$ & $\begin{array}{l}\text { - Délai d'apparition de la 1 1̀re } \\
\text { exacerbation } \\
\text { - VEMS post } \\
\text { bronchodilatateur } \\
\text { - TM6 } \\
\text { - Effets secondaires }\end{array}$ & $\begin{array}{l}\text { - Placebo } \mathrm{n}=70: 1,57 \\
\text { exacerbations par patient par an } \\
\text { - AZM } \mathrm{n}=71: 0,59 \text { exacerbations } \\
\text { par patient par an }\end{array}$ & $\begin{array}{l}\text {-Placebo : } \\
1^{\text {ere }} \text { exacerbation : J21 } \\
\text {-AZM : } \\
1^{\text {ere }} \text { exacerbation : } \\
\text { J104 } \\
\text { Pas de différences sur } \\
\text { les autres critères }\end{array}$ \\
\hline $\begin{array}{l}\text { Altenbourg et } \\
\text { al. } \\
\text { (9) }\end{array}$ & $\begin{array}{l}\text { Multicentrique } \\
\text { Randomisée } \\
\text { Contrôlée } \\
\text { Double aveugle } \\
89 \text { patients }\end{array}$ & $\begin{array}{l}\text { AZM } \\
250 \mathrm{mg} \text { par jour }\end{array}$ & 1 an & $\begin{array}{l}\text { Au moins } 3 \text { exacerbations } \\
\text { l'année précédente }\end{array}$ & $\begin{array}{l}\text { Nombre d'exacerbations } \\
\text { pendant la durée de l'étude }\end{array}$ & $\begin{array}{l}\text { - spirométrie } \\
\text { - qualité de vie } \\
\text { - CRP } \\
\text { - effets secondaires }\end{array}$ & $\begin{array}{l}\text { - placebo } n=43: 2,05 \\
\text { exacerbations par patient par an } \\
\text { - AZM } n=40: 0,84 \\
\text { exacerbations par patient par an } \\
P<0,0001\end{array}$ & $\begin{array}{l}\text { Tendance à } \\
\text { l'amélioration de } \\
\text { VEMS : }+1,03 \% \text { tous } \\
\text { les } 3 \text { mois (AZM) vs - } \\
0,1 \% \text { (placebo) }\end{array}$ \\
\hline
\end{tabular}

AZM : azithromycine; CRP : C-réactive protéine; VEMS : volume expiratoire maximale seconde; ATB : antibiotique; TM6 : test de marche six minutes; SGRQ : Saint George's respiratory pulmonaire ; DDB : dilatation de bronche 
Tableau 3 : Principales caractéristiques des études dans l'asthme.

\begin{tabular}{|c|c|c|c|c|c|c|c|c|}
\hline Auteur & Méthodologie & Intervention & Durée & Critère d'inclusion & $\begin{array}{l}\text { Critère de jugement } \\
\text { principal }\end{array}$ & Critère secondaires & $\begin{array}{l}\text { Résultats critères de jugement } \\
\text { principal }\end{array}$ & $\begin{array}{l}\text { Résultats sur } \\
\text { critère secondaires }\end{array}$ \\
\hline $\begin{array}{l}\text { Brusselle } \text { et al. } \\
\text { (11) }\end{array}$ & $\begin{array}{l}\text { Randomisée } \\
\text { Double aveugle } \\
\text { Contre placebo } \\
\mathrm{N}=109 \text { patients }\end{array}$ & $\begin{array}{l}\text { AZM } 250 \mathrm{mg} \\
3 \text { jours par semaine }\end{array}$ & 6 mois & $\begin{array}{l}\text { Asthme sévère } \\
\text { Exacerbateurs }\end{array}$ & Taux d'exacerbation & $\begin{array}{l}\text { - Fonction pulmonaire } \\
\text { - ACQ } \\
\text { - AQLQ } \\
\text { - Tolérance }\end{array}$ & $\begin{array}{l}\text { - } \mathrm{N}=55 \text { AZM taux exacerbation : } 0,75 \\
\text { par patient par an } \\
\text { - N=54 placebo, nombre } \\
\text { exacerbation : } 0,81 \text { par patient par an } \\
\mathrm{p}=0,682, \text { mais significatif dans le } \\
\text { groupe asthme non éosinophile } \\
\text { (nombre exacerbation } 0,44 \text { versus } \\
1,03 \mathrm{p}=0,013 \text { ) }\end{array}$ & $\begin{array}{l}\text { Pas de différences } \\
\text { significatives sur les } \\
\text { critères secondaires }\end{array}$ \\
\hline $\begin{array}{l}\text { Gibson et al. } \\
\text { (12) }\end{array}$ & $\begin{array}{l}\text { Randomisée } \\
\text { Double aveugle } \\
\text { Contrôlé } \\
\text { Contre placebo } \\
\mathrm{N}=420 \text { patients }\end{array}$ & $\begin{array}{l}\text { AZM } 500 \mathrm{mg} \\
3 \text { jours par semaine }\end{array}$ & $\begin{array}{l}-48 \\
\text { semaines }\end{array}$ & $\begin{array}{l}\text { Asthme non } \\
\text { contrôlé malgré } \\
\text { traitement usuel }\end{array}$ & Taux d'exacerbation & $\begin{array}{l}\text { - ACQ } \\
\text { - Fonction pulmonaire } \\
\text { - Tolérance } \\
\text { - Données } \\
\text { bactériologiques }\end{array}$ & $\begin{array}{l}\text { - N=213 AZM } \\
\text { Nombre d'exacerbation : 1,07 par } \\
\text { année par patient } \\
\text { - N=207 placebo } \\
\text { Nombre d'exacerbation : } 1,86 \text { par } \\
\text { année par patient } \\
\text { p }<0,0001\end{array}$ & $\begin{array}{l}\text { Pas de différences } \\
\text { significatives sur les } \\
\text { critères secondaires }\end{array}$ \\
\hline
\end{tabular}

ACQ : asthma control questionnaire, AZM : azithromycine, AQLQ : asthma qualitiy of life questionnaire 